\title{
Hemodynamic response to primary prophylactic therapy with nonselective $\beta$-blockers is related to a reduction of first variceal bleeding risk in liver cirrhosis: a meta-analysis
}

\author{
Annarein J.C. Kerbert ${ }^{a, \star}$, Fang W.T. Chiang ${ }^{a, \star}$, Mark van der Werf ${ }^{b}$, Theo Stijnen ${ }^{b}$, Hilde Slingerland ${ }^{a}$, \\ Hein W. Verspaget ${ }^{a}$, Bart van Hoek ${ }^{a}$ and Minneke J. Coenraad ${ }^{a}$
}

\begin{abstract}
The current primary prophylaxis for esophageal variceal bleeding in cirrhotic patients consists of nonselective $\beta$-blocker (NSBB) therapy. However, only approximately half of the patients achieve a sufficient hemodynamic response to NSBB therapy. Clinical application of hemodynamic response monitoring is still under debate. The aim of this meta-analysis is to assess the potential clinical value of monitoring the hemodynamic response to NSBB therapy using hepatic venous pressure gradient (HVPG) measurements in the primary prophylaxis for variceal bleeding. A systematic literature search was performed in PubMed, Embase, Web of Science, and the COCHRANE Library. Randomized-controlled trials and case series that included cirrhotic patients receiving primary prophylaxis for variceal bleeding with NSBBs and hemodynamic response monitoring using HVPG measurements were included for analysis. The primary outcome measure was variceal bleeding. A fixed-effect analysis was carried out using the Mantel-Haenszel method for relative risks. Six of the 1172 papers found were selected on the basis of stringent selection criteria. Hemodynamic response (HVPG $\leq 12 \mathrm{mmHg}$ and/or a reduction of $\geq 20 \%$, or $\geq 10 \%$ in one study, from baseline) to $\beta$-blocker therapy was associated significantly with a lower risk of variceal bleeding (relative risk $=0.13,95 \%$ confidence interval $=0.06-0.29$ ) compared with a nonresponse. Patients achieving a hemodynamic response to NSBB therapy have a lower risk of variceal bleeding than hemodynamic nonresponders. Hemodynamic monitoring in primary prophylaxis is of potential clinical value and requires further assessment in large cohort randomized-controlled trials. Eur J Gastroenterol Hepatol 29:380-387

Copyright (O) 2017 Wolters Kluwer Health, Inc. All rights reserved.
\end{abstract}

\section{Introduction}

Esophageal varices are common in cirrhotic patients with portal hypertension with a prevalence of $\sim 50 \%$ [1]. Acute variceal bleeding occurs at an annual rate of $5-15 \%$ per year and is the most common lethal complication in cirrhosis. It is associated with a 6 -week mortality rate of $20 \%$ [1] and with rebleeding rates of $60-70 \%$ within the first 2 years in the absence of secondary prophylactic treatment [2]. Therapeutic options to prevent a first bleeding episode include pharmacological therapy with nonselective $\beta$ blockers (NSBBs), endoscopic band ligation, and placement of transjugular intrahepatic portosystemic shunts [3].

\footnotetext{
European Journal of Gastroenterology \& Hepatology 2017, 29:380-387

Keywords: cirrhosis, hepatic venous pressure gradient, nonselective $\boldsymbol{\beta}$-blockers, primary prophylaxis, variceal bleeding

Departments of a Gastroenterology and Hepatology and bedical Statistics and Bio-Informatics, Leiden University Medical Center, Leiden, The Netherlands

Correspondence to Annarein J.C. Kerbert, BSc, Department of Gastroenterology and Hepatology, Leiden University Medical Center, Albinusdreef 2, 2333 ZA Leiden, The Netherlands

Tel: + 316410 12403; fax: + 3171524 8115; e-mail: j.c.kerbert@lumc.nl

*Annarein J.C. Kerbert and Fang W.T. Chiang contributed equally to the writing of this article.

Received 1 September 2016 Accepted 4 November 2016

Supplemental digital content is available for this article. Direct URL citations appear in the printed text and are provided in the HTML and PDF versions of this article on the journal's website (www.eurojgh.com).
}

Primary prophylaxis with NSBBs is currently recommended as the standard of care in cirrhotic patients with medium-large varices and patients with small varices who are at an increased risk of bleeding [4]. NSBBs reduce portal pressure by decreasing cardiac output and producing splanchnic vasoconstriction. Treatment with NSBBs decreases the risk of a first bleeding episode by $\sim 50 \%$ in various studies $[5,6]$. However, reduction of the heart frequency by NSBB therapy and reduction in portal venous pressure are not correlated [7]. Measurement of the hepatic venous pressure gradient (HVPG) is currently the most commonly used method to determine the presence and severity of portal hypertension [8-10]. An HVPG of at least $10 \mathrm{mmHg}$ is considered clinically significant portal hypertension at which complications such as the development of esophageal varices may occur. Various studies have shown that a reduction of the HVPG up to $12 \mathrm{mmHg}$ or of at least $10-20 \%$ from baseline is associated with a significantly decreased risk of a variceal bleeding episode [11-13]. However, only 50\% of patients treated with NSBBs achieve this hemodynamic response [14]. HVPG measurement can guide prophylactic therapy by identification of hemodynamically nonresponding patients to provide add-on or alternative therapy in this subgroup $[14,15]$. The clinical application of HVPG measurement in the prevention of variceal bleeding with NSBBs is still under debate [16-20]. A previous meta-analysis [14] and systematic review [16] showed that a reduction of the HVPG 
provides valuable prognostic information in the setting of primary and secondary prophylaxis for variceal bleeding. More recently, the use of HPVG was recommended in the Baveno VI consensus [4]. In clinical practice, HVPG monitoring of primary prophylactic therapy with NSBBs is not widely implemented because of the lack of evidence, experience, and costs. The aim of this meta-analysis is to assess the prognostic value of monitoring the hemodynamic response to NSBB therapy using HVPG measurements in primary prophylaxis for variceal bleeding in cirrhotic patients.

\section{Materials and methods}

\section{Search strategy}

A systematic literature search was performed to identify eligible studies (date of the search: 9 March 2016). Assisted by a trained librarian, a search was performed in the following databases: PubMed, Embase (OVID version), Web of Science, and the COCHRANE Library. The subject query was applied to all databases taking into account the terminological differences between them. The query consisted of the following subjects: 'liver cirrhosis', 'esophageal varices', 'blood pressure', ' $\beta$-blockers', and 'prophylaxis'. Various synonyms and related terms for all subjects were used in the search (Supplementary Table 1, Supplemental digital content 1, http://links.lww.com/ EJGH/A159). For the articles included, an additional citation tracking search was performed for unique references that were not found in the initial systemic search.

\section{Study selection}

Titles, abstracts, and full articles were screened independently for eligibility by two authors. Disagreement between reviewers was resolved through consensus. Exclusion criteria were as follows: studies unrelated to variceal bleeding, pediatric and experimental studies, studies on secondary prevention (i.e. patients with a previous variceal bleeding episode), and study designs other than randomized-controlled trials and prospective case series. Conference abstracts were also included in the literature search. In case of duplicate data or results published in multiple studies, the most recent and/or complete data were included for data extraction and analysis. Specific reasons for excluding studies are shown in Fig. 1. Study quality of the nonrandomized studies selected for metaanalysis was assessed using the Newcastle-Ottawa Scale for observational studies [21]. This scale is designed to assess the risk of bias in three domains: patient selection, comparability of study groups, and the ascertainment of either the exposure or outcome of interest for case-control studies or cohort studies, respectively.

\section{Data extraction}

The following data were extracted from the studies included: study design, number of included and evaluated patients, etiology of cirrhosis, Child-Pugh score, specific type of prophylaxis used, hemodynamic response to therapy, number of variceal bleeding episodes (subdivided on the basis of hemodynamic response), mean value of baseline HVPG, time to remeasurement, and follow-up time.
Hemodynamic response was defined as a reduction in HVPG up to $12 \mathrm{mmHg}$ or of at least $20 \%$ from baseline in all studies. One study [22] applied a cut-off value of an HVPG up to $12 \mathrm{mmHg}$ or a decrease of at least $10 \%$ from baseline. We applied the definitions of hemodynamic response identical to those applied in the individual selected studies as described above.

\section{Statistical analysis}

The primary outcome measure was a first variceal bleeding episode during follow-up. The number of first variceal bleeding episodes was recorded for both the responder and the nonresponder group. The pooled relative risk (RR) with a $95 \%$ confidence interval (CI) for responders versus nonresponders was estimated by meta-analysis using fixedeffect and random-effects models. Statistical heterogeneity of the studies was evaluated to determine whether to apply a fixed-effect or a random-effects model. The heterogeneity was estimated by calculating the residual between-trial variance $\tau$ using a restricted maximum likelihood method. The estimated $\tau$ was 0.00 and the Cochrane $\chi^{2}$-test indicated no significant heterogeneity $(P=0.77)$. Therefore, the random-effects model was reduced to a fixed-effects model. The model was fitted using the natural $\log$ of the risk ratios of the individual studies, which were weighed using inverse variance. As the assumption of normality of the log risk ratios could be violated, an additional fixedeffects analysis was carried out using the Mantel-Haenszel method. This method is less biased and especially more advantageous in small sample sizes, which is the case for the studies included [23].

\section{Results}

\section{Literature search}

The bibliographic databases yielded a total of 1172 references, which were screened for eligibility. In total, 977 abstracts were assessed after title screening. Editorials, letters, reviews, overviews, opinions, and case reports were excluded from further assessment. Abstracts unrelated to pharmacological prophylaxis of variceal bleeding were also excluded. 104 full articles were selected for a detailed review. The reasons for the exclusion of 98 of these articles are shown in Fig. 1. One study [24] that fulfilled the selection criteria was excluded from meta-analysis because of the small study population and the absence of bleeding events in both the responder and the nonresponder group. Finally, six peer-reviewed articles, including a total of 308 patients, were selected for the final analysis (Fig. 1). An additional 287 unique articles were found through citation tracking of these included articles, but of these, no eligible papers were selected after screening.

\section{Study characteristics}

The study characteristics of the selected papers are outlined in Tables 1 and 2. The selected papers included one randomized-controlled trial [12] and five prospective nonrandomized case series [15,22,25-27]. The mean Newcastle-Ottawa Scale score was 7.6 (range: 7-8) for the five nonrandomized studies (Supplementary Table 2, Supplemental digital content 2, http://links.lww.com/EJGH/A159). 


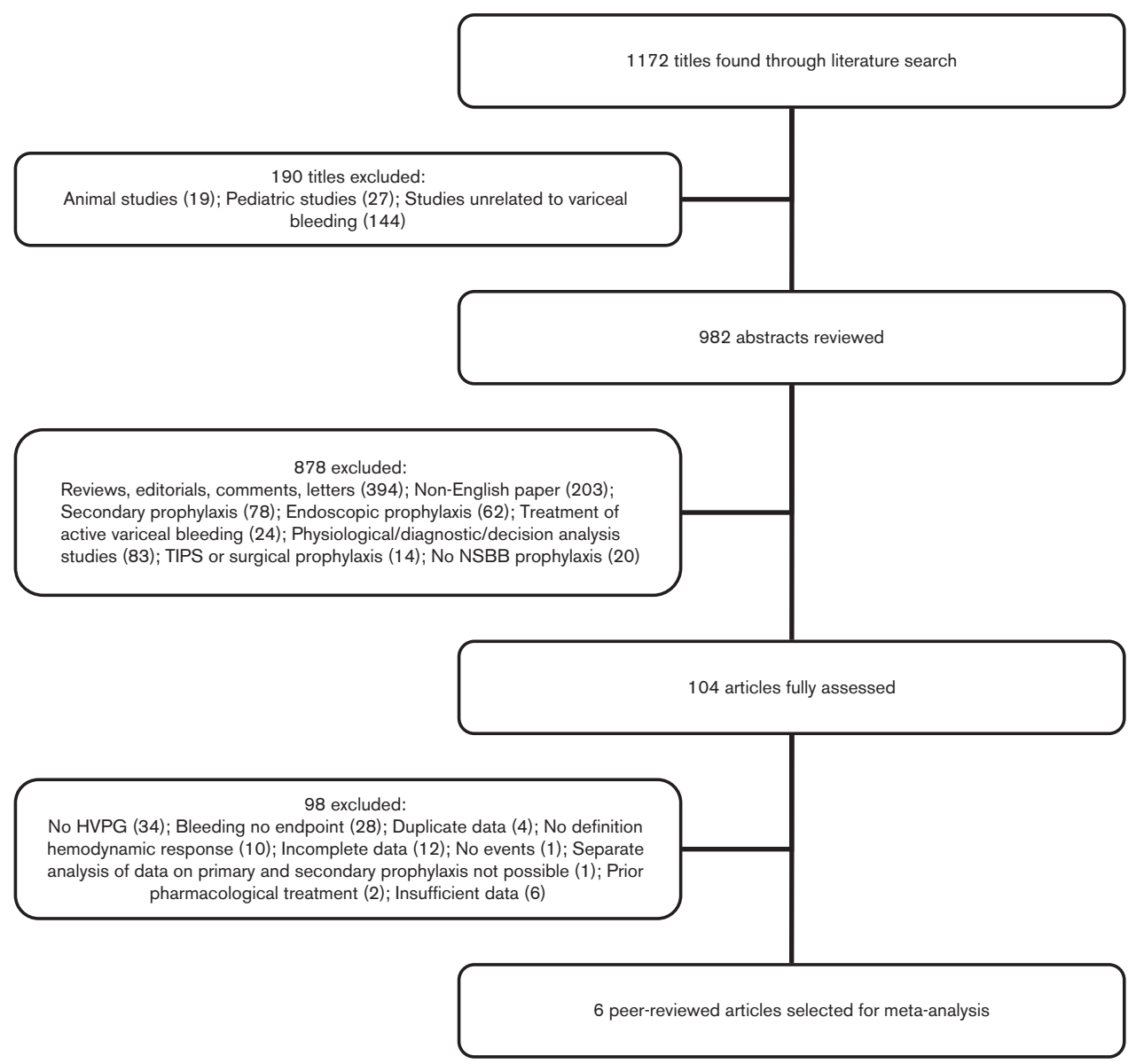

Fig. 1. Flowchart showing the selection process of the retrieved studies for meta-analysis of hemodynamic monitoring of pharmacological primary prophylaxis for variceal bleeding in cirrhosis. HVPG, hepatic venous pressure gradient; NSBB, nonselective $\beta$-blocker; TIPS, transjugular intrahepatic portosystemic shunt.

All patients $(n=414)$ were treated with NSBBs, either propranolol or nadolol. In two of the studies, organic nitrates were added if patients did not achieve the target hemodynamic response to NSBBs alone [15,25]. In these studies, a total of 39 patients received organic nitrates; of these, $10(25.6 \%)$ patients ultimately achieved a hemodynamic response. These patients were analyzed in the hemodynamic response group together with patients who responded to NSBB monotherapy. In the study of Merkel et al. [25], 20 patients received organic nitrates irrespective of the presence of a hemodynamic response.

Classification of varices was variable between the trials. Two studies included patients with varices irrespective of their size or classification [12,26]. Villanueva et al. [22] only included patients with large varices (i.e. $\geq 5 \mathrm{~mm}$ ), whereas Merkel et al. [25] included patients with both large varices and small varices (i.e. $<5 \mathrm{~mm}$ ) with red whale signs. Bureau et al. [15] and Sharma et al. [27] included only patients with varices of grade II or III and grade III or IV, respectively.

The time interval between the first and second HVPG measurement differed among the studies included. Four studies carried out the second measurement at $\sim 1$ month after baseline measurement, whereas two studies repeated the HVPG measurement after 3 and 4 months, respectively (Table 2).

\section{Variceal bleeding and hemodynamic response}

The correlation between variceal bleeding and hemodynamic response to NSBBs was calculated by combining the data of the six studies. Table 3 summarizes the proportions of variceal bleeding episodes in relation to the development of a hemodynamic response on primary prophylaxis in the individual studies. Of all 414 patients included in the six selected studies, $308(74.4 \%)$ patients were evaluated in the final meta-analysis assessing the relative risk for a first variceal bleeding episode in hemodynamic responders versus nonresponders to NSBB treatment. Specifications of reasons for excluding subgroups of patients from the final meta-analysis as reported by the authors of the six included studies are shown in Supplementary Table 3, Supplemental digital content 3, http://links.lww.com/ EJGH/A159. In total, 157 out of the 308 (50.9\%) evaluated patients achieved a hemodynamic response to pharmacological prophylaxis. The proportion of bleeding episodes in the hemodynamic response group was six out of $157(3.8 \%)$ patients. In the nonresponding group, 42 out of 151 [27.8\%, (Table 3)] patients had a bleeding episode. The occurrence of a bleeding episode during follow-up in the subgroups of patients treated with propranolol in combination with organic nitrates was not specified in the respective studies. 


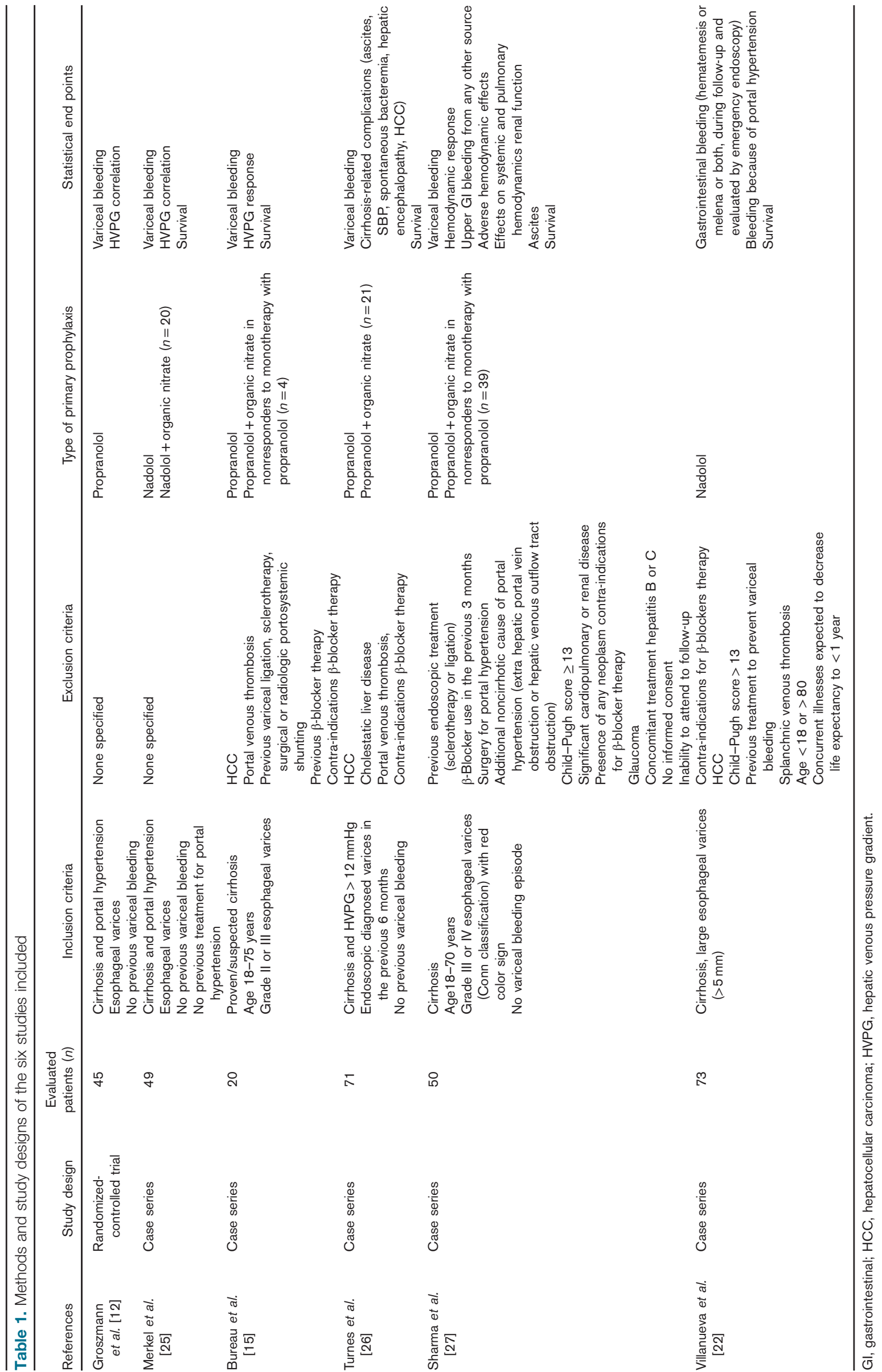

Copyright (C) 2017 Wolters Kluwer Health, Inc. All rights reserved. 

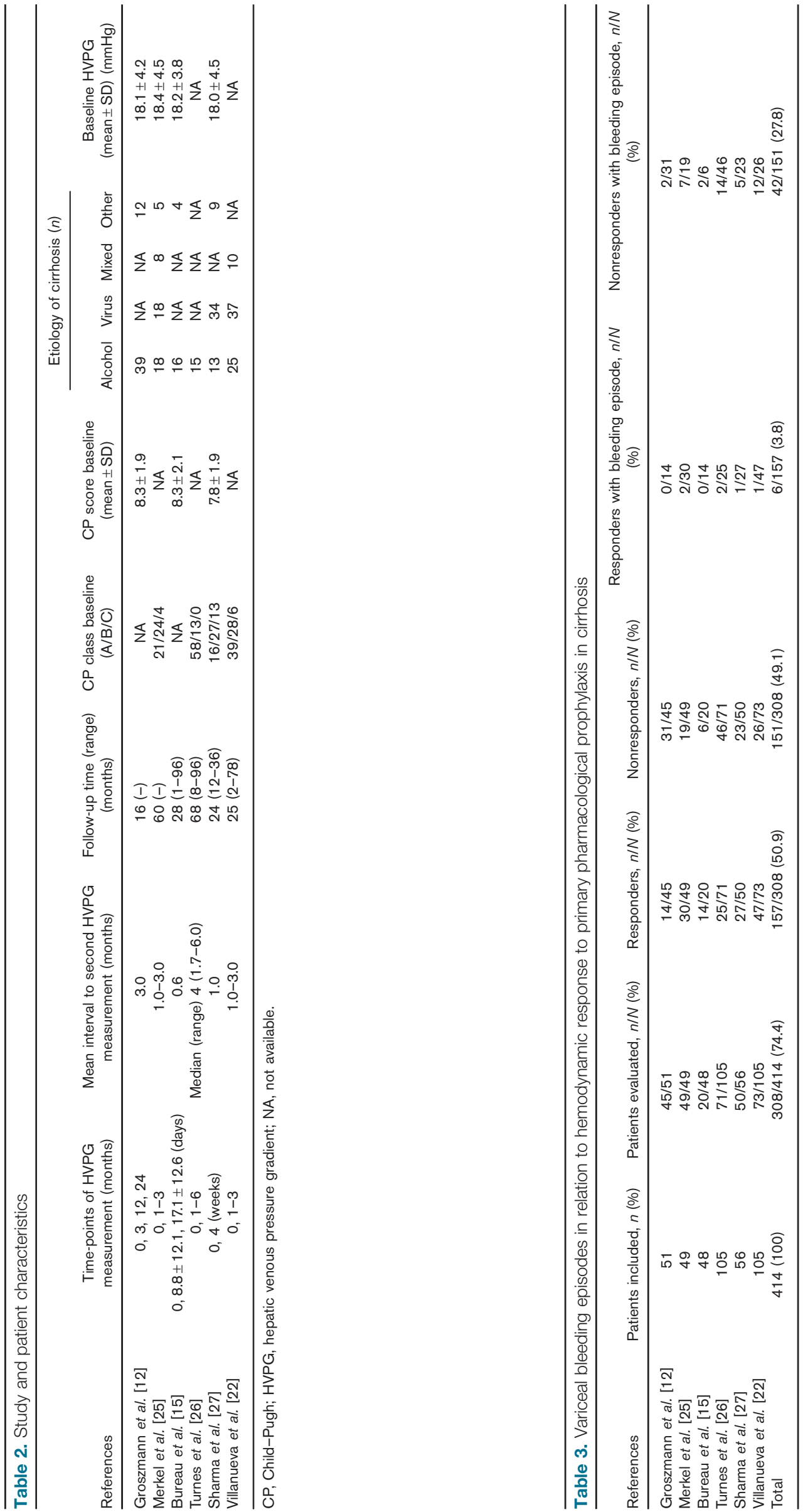

Copyright (C) 2017 Wolters Kluwer Health, Inc. All rights reserved. 


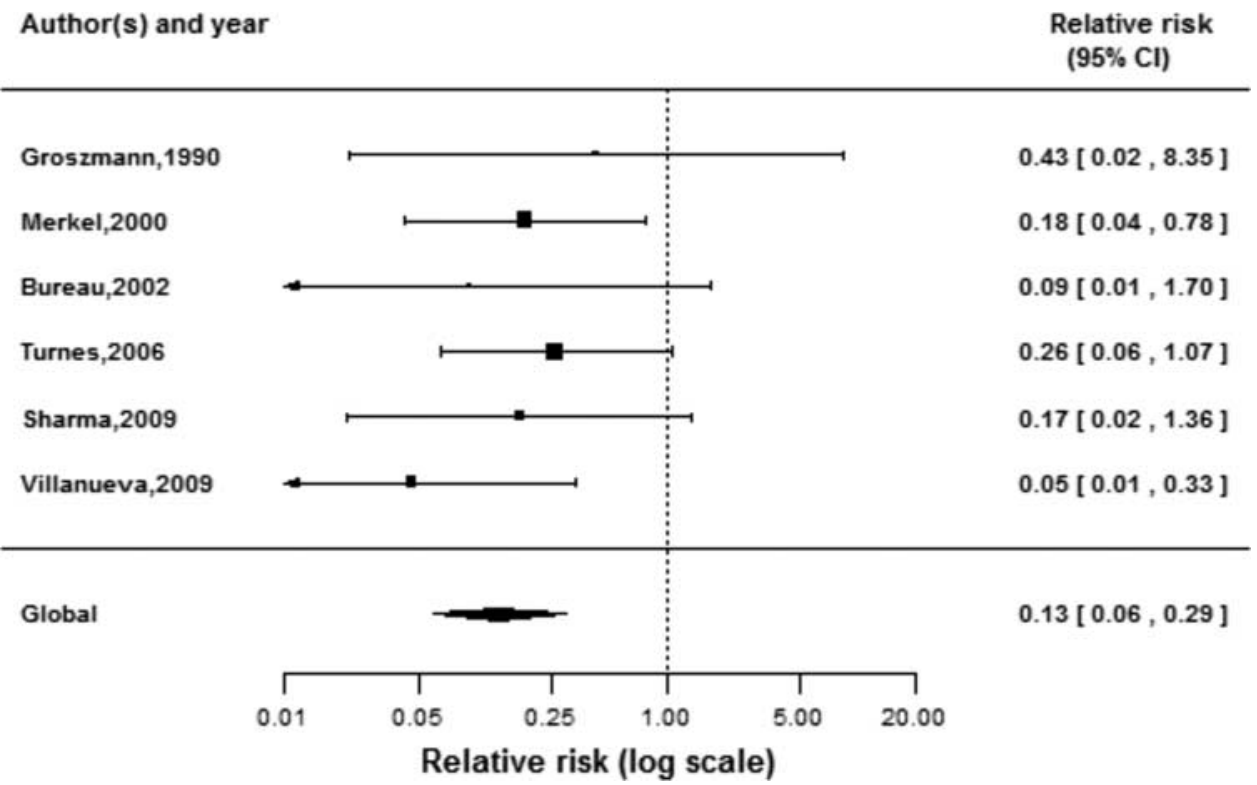

Fig. 2. Forest plot Mantel-Haenszel fixed-effects model for the relative risk of variceal bleeding in hemodynamic responders on primary prophylaxis with nonselective $\beta$-blocker therapy. $\mathrm{Cl}$, confidence interval.

The RR of bleeding in hemodynamic responders compared with nonresponders was 0.13 (95\% CI: 0.06-0.29) through a fixed-effect analysis with the Mantel-Haenszel method (Fig. 2). No significant heterogeneity in RR was found between the studies $(P=0.77)$. Applying the definition of at least $20 \%$ HVPG reduction from baseline also for the study of Villanueva et al. [22], the RR of bleeding in hemodynamic responders became 0.14 (95\% CI: $0.05-0.35)$. Conditional logistic regression showed that the RR was not dependent on the median time to remeasurement $(P=0.39)$.

\section{Discussion}

Portal hypertension in liver cirrhosis is a progressive condition, leading to the development of esophageal varices at a rate of $\sim 7 \%$ per year $[28,29]$. HVPG measurement is the gold-standard method to assess the presence of portal hypertension [4]. The threshold HVPG value at which gastroesophageal varices develop is $10 \mathrm{mmHg}$. These patients are referred to as having clinically significant portal hypertension. A noninvasive assessment of clinically significant portal hypertension by measuring liver stiffness using transient elastography is currently only considered sufficient in patients with viral-related chronic liver disease. In addition, the degree of liver stiffness can, together with platelet count, predict the risk of the presence of gastroesophageal varices. Therefore, transient elastography can also be used to identify patients who should undergo screening esophagogastroduodenoscopy [4]. The risk of bleeding from esophageal varices is largely dependent on their size, which is the most commonly used factor in clinical practice to identify patients who are at a high risk of bleeding. Patients with small varices $(<5 \mathrm{~mm})$ have a bleeding risk of $\sim 12 \%$ in the first 2 years after diagnosis compared with $30 \%$ in patients with medium-large varices ( $\geq 5 \mathrm{~mm}$ ) [29-31]. Other important risk factors for variceal bleeding include the severity of the underlying liver disease, as defined by the Child-Pugh score, and the presence of red wale marks on the variceal wall [32]. To improve the ability of risk assessment for variceal bleeding, several other factors have been explored, such as variceal wall tension [33] and portal pressure [34,35], as reflected by the HVPG. It has been found that the level of the HVPG significantly improves the prediction of variceal bleeding risk $[35,36]$. Primary prophylaxis with NSBBs, which is the first-choice treatment option [4], is based on its ability to decrease portal blood flow by reducing cardiac output and inducing splanchnic vasoconstriction, resulting in decreased variceal wall tension. It has been proven that NSBB therapy significantly reduces the risk of a first bleeding episode compared with nontreated patients: $\sim 20$ versus $\sim 35 \%$ bleeding risk within 2 years of diagnosis $[14,37]$. NSBB therapy achieves a target reduction of the HVPG up to $12 \mathrm{mmHg}$ or a reduction of more than $20 \%$ from baseline in $\sim 50 \%$ of patients [14]. A previous metaanalysis by Albillos et al. [14] showed that hemodynamic responders are at a significantly lower risk of developing a first bleeding episode than nonresponders (6 vs. $32 \%$ ). This was confirmed in the present meta-analysis, which included more recent data and a higher number of study participants compared with the previous meta-analysis. These findings imply that it might be clinically relevant to monitor the hemodynamic response on NSBBs using repeated HVPG measurements and providing additional therapy, such as endoscopic band ligation, to nonresponding patients. However, no studies investigating the cost-effectiveness of this strategy have been carried out so far.

Some heterogeneity in the trial-specific characteristics of studies included in the present meta-analysis needs to be considered. Firstly, the time interval between remeasurement of the HVPG differed between the studies. It has been suggested previously that an early hemodynamic response is associated with a higher ability of predicting variceal bleeding in the long term compared with a late response 
[14]. In addition, it has been found that the hemodynamic response to NSBBs changes after long-term follow-up in about $20 \%$ of patients [38]. However, in the present study, no correlation was found between bleeding risk and the median time to the second HVPG measurement. Therefore, we could not identify the optimal time interval for remeasurement on the basis of these data. Second, different techniques of HVPG measurement were used in the studies included. Two major techniques are used in clinical practice: the straight catheter and the balloontipped catheter technique. Both methods are considered safe and reproducible procedures [8,9], but studies have shown that the balloon-tipped catheter technique is more accurate in estimating portal pressure compared with the straight catheter $[39,40]$. Although five out of six studies used the balloon-tipped catheter technique, there were differences in material brands and accessed vein. To minimize the potential effect of the technique and the type of materials used, standardization of these factors in future studies should be applied. Finally, the studies included used different inclusion criteria with respect to variceal size, which is a major determinant for variceal bleeding risk. Despite these differences in variceal classification, no significant heterogeneity in RR for the risk of a first variceal bleeding episode was found between the studies. The mean baseline HVPG was $\sim 18 \mathrm{mmHg}$ in all the studies included, which therefore mainly assessed the hemodynamic response rate in clinically significant portal hypertension. A recent study by Villanueva et al. [41] showed that patients with subclinical portal hypertension have significantly lower portal pressure reduction after acute $\beta$ blockade than patients with clinical significant portal hypertension, suggesting that NSBB therapy is more effective in the former subgroup. Associations with bleeding risk of other relevant trial-level covariates, such as severity and etiology of cirrhosis, follow-up time, and treatment dose, could not be analyzed in this meta-analysis because individual patient data were not described in all papers. The effect of a hemodynamic response to NSBB prophylaxis on liver-related mortality was not assessed in all studies either.

Currently, traditional NSBBs (i.e. propranolol and nadolol) as well as carvedilol are recommended for primary prophylactic therapy [4]. Carvedilol seems to be more effective in decreasing HVPG than traditional NSBBs [42] and switching to carvedilol in nonresponders to propranolol may result in the achievement of a hemodynamic response [43]. However, adequate comparison in clinical trials is still lacking. As none of the studies included used carvedilol as primary prophylactic therapy, we could not compare the effect of these $\beta$-blocker types either. Various add-on therapies have been studied to support NSBB therapy, such as vasodilators, diuretics, statins, and organic nitrates. Nevertheless, sufficient evidence for implementation of these therapies in clinical practice is lacking. Addition of organic nitrates to NSBB therapy has been found to increase the rate of responders by one-third [44]. However, a previous meta-analysis showed a nonsignificant lower bleeding rate, similar mortality rates, and more adverse effects in the group of patients receiving combined prophylactic therapy compared with patients treated with NSBBs alone $[25,45,46]$. In the present metaanalysis, combined therapy with NSBBs and organic nitrates was performed in four studies. In two of these studies, organic nitrates were added because of a hemodynamic nonresponse to NSBBs, resulting in an additional hemodynamic response rate of $25.6 \%$. However, differences in the variceal bleeding risk between the monotherapy and the combination therapy group were not reported.

\section{Conclusion}

Hemodynamic monitoring in primary prophylaxis seems to be a strong prognostic tool in risk assessment for variceal bleeding in cirrhosis. The ideal add-on therapy has not been established as yet. According to the Baveno VI consensus, measurement of HVPG response to treatment offers relevant additional information. The costeffectiveness of hemodynamic monitoring and impact on variceal bleeding incidence should be further explored in future randomized-controlled trials.

\section{Acknowledgements}

Research grant from the Leiden University Medical Center, Leiden, the Netherlands (grant number: 8219-70550).

\section{Conflicts of interest}

There are no conflicts of interest.

\section{References}

1 Garcia-Tsao G, Sanyal AJ, Grace ND, Carey W. Prevention and management of gastroesophageal varices and variceal hemorrhage in cirrhosis. Hepatology 2007; 46:922-938.

2 Burroughs AK, McCormick PA. Natural history and prognosis of variceal bleeding. Baillieres Clin Gastroenterol 1992; 6:437-450.

3 Bureau C. How to prevent first variceal bleeding? Gastroenterol Clin Biol 2004; 28:B44-B52.

4 De Franchis R. on behalf of the Baveno VI Faculty. Expanding consensus in portal hypertension. Report of the Baveno VI Consensus workshop: stratifiying risk and individualizing care for portal hypertension. J Hepatol 2015; 63:743-752.

5 Poynard T, Cales P, Pasta L, Ideo G, Pascal JP, Pagliaro L, Lebrec D. Beta-adrenergic-antagonist drugs in the prevention of gastrointestinal bleeding in patients with cirrhosis and esophageal varices. An analysis of data and prognostic factors in 589 patients from four randomized clinical trials. Franco-Italian Multicenter Study Group. N Engl J Med 1991; 324:1532-1538.

6 D'Amico G, Pagliaro L, Bosch J. The treatment of portal hypertension: a meta-analytic review. Hepatology 1995; 22:332-354.

7 Garcia-Tsao G, Grace ND, Groszmann RJ, Conn HO, Bermann MM, Patrick MJ, et al. Short-term effects of propranolol on portal venous pressure. Hepatology 1986; 6:101-106.

8 Parikh S. Hepatic venous pressure gradient: worth another look? Dig Dis Sci 2009; 54:1178-1183.

9 Kumar A, Sharma P, Sarin SK. Hepatic venous pressure gradient measurement: time to learn!. Indian J Gastroenterol 2008; 27:74-80.

10 Groszmann R, Vorobioff JD, Gao H. Measurement of portal pressure: when, how, and why to do it. Clin Liver Dis 2006; 10:499-512.

11 Groszmann RJ, Garcia-Tsao G, Bosch J, Grace ND, Burroughs AK, Planas R, et al. Portal Hypertension Collaborative Group. Beta-blockers to prevent gastroesophageal varices in patients with cirrhosis. N Engl J Med 2005; 353:2254-2261.

12 Groszmann RJ, Bosch J, Grace ND, Conn HO, Garcia-Tsao G, Navasa M, et al. Hemodynamic events in a prospective randomized trial of propranolol versus placebo in the prevention of a first variceal hemorrhage. Gastroenterology 1990; 99:1401-1407.

13 Feu F, Garcia-Pagan JC, Bosch J, Luca A, Terés J, Escorsell A, Rodés J. Relation between portal pressure response to pharmacotherapy and risk of recurrent variceal haemorrhage in patients with cirrhosis. Lancet 1995; 346:1056-1059. 
14 Albillos A, Bañares R, González M, Ripoll C, Gonzalez R, Catalina MV, Molinero LM, et al. Value of the hepatic venous pressure gradient to monitor drug therapy for portal hypertension: a meta-analysis. $A m \mathrm{~J}$ Gastroenterol 2007; 102:1116-1126.

15 Bureau C, Peron JM, Alric L, Morales J, Sanchez J, Barange K, et al. 'A La Carte' treatment of portal hypertension: adapting medical therapy to hemodynamic response for the prevention of bleeding. Hepatology 2002; 36:1361-1366.

16 D'Amico G, Garcia-Pagan JC, Luca A, Bosch J. Hepatic vein pressure gradient reduction and prevention of variceal bleeding in cirrhosis: a systematic review. Gastroenterology 2006; 131:1611-1624.

17 Thalheimer U, Mela M, Patch D, Burroughs AK. Targeting portal pressure measurements: a critical reappraisal. Hepatology 2004; 39:286-290.

18 Sanyal AJ. Hepatic venous pressure gradient: to measure or not to measure, that is the question. Hepatology 2000; 32:1175-1176.

19 Merkel C, Montagnese S. Should we routinely measure portal pressure in patients with cirrhosis, using hepatic venous pressure gradient (HVPG) as guidance for prophylaxis and treatment of bleeding and rebleeding? Yes! Eur J Intern Med 2011; 22:1-4.

20 Thalheimer U, Bellis L, Puoti C, Burroughs AK. Should we routinely measure portal pressure in patients with cirrhosis, using hepatic venous pressure gradient (HVPG) as a guide for prophylaxis and therapy of bleeding and rebleeding? No. Eur J Intern Med 2011; 22:5-7.

21 Wells G, Shea B, O'Connell D, Peterson J, Welch V, Losos M, Tugwell P. The Newcastle-Ottawa (NOS) for assessing the quality of nonrandomized studies in a meta-analysis. Ottawa Hospital Research Institute. http://www.ohri.ca/programs/clinical-epidemiology/oxford.htm. [Accessed 27 October 2016].

22 Villanueva C, Aracil C, Colomo A, Hernández-Gea V, LópezBalaguer JM, Alvarez-Urturi C, et al. Acute hemodynamic response to beta-blockers and prediction of long-term outcome in primary prophylaxis of variceal bleeding. Gastroenterology 2009; 137:119-128.

23 Higgins JPT, Green S. Cochrane handbook for systematic reviews of interventions version 510. The Cochrane Collaboration; 2011. Available at: http://handbook.cochrane.org. [Accessed 8 July 2016].

24 de-Madaria E, Palazon JM, Hernandez FT, Sánchez-Paya J, Zapater P, Irurzun $\mathrm{J}$, et al. Acute and chronic hemodynamic changes after propranolol in patients with cirrhosis under primary and secondary prophylaxis of variceal bleeding: a pilot study. Eur J Gastroenterol Hepatol 2010; 22:507-512

25 Merkel C, Bolognesi M, Sacerdoti D, Bombonato G, Bellini B, Bighin B, Gatta $A$. The hemodynamic response to medical treatment of portal hypertension as a predictor of clinical effectiveness in the primary prophylaxis of variceal bleeding in cirrhosis. Hepatology 2000; 32:930-934.

26 Turnes J, Garcia-Pagan JC, Abraldes JG, Hernandez-Guerra M, Dell'Era A, Bosch J. Pharmacological reduction of portal pressure and long-term risk of first variceal bleeding in patients with cirrhosis. Am J Gastroenterol 2006; 101:506-512.

27 Sharma P, Kumar A, Sharma BC, Sarin SK. Early identification of haemodynamic response to pharmacotherapy is essential for primary prophylaxis of variceal bleeding in patients with 'high-risk' varices. Aliment Pharmacol Ther 2009; 30:48-60.

28 Merli M, Nicolini G, Angeloni S, Rinaldi V, De Santis A, Merkel C, et al. Incidence and natural history of small esophageal varices in cirrhotic patients. J Hepatol 2003; 38:266-272.

29 Pagliaro L, D’Amico G, Pasta L, Politi F, Vizzini G. Traina M. Portal hypertension in cirrhosis: natural history. In: Bosch J, Groszmann RJ, editors. Portal hypertension: pathophysiology and treatment. Oxford: Blackwell Science; 1994. pp. 72-92.
30 Madhotra R, Mulcahy HE, Willner I, Reuben A. Prediction of esophageal varices in patients with cirrhosis. J Clin Gastroenterol 2002; 34:81-85.

31 D'Amico G. Esophageal varices: from appearance to rupture: natural history and prognostic indicators. In: Groszmann RJ, Bosch J, editors. Portal hypertension in the 21st century. Dortrecht, the Netherlands: Kluwer Academia Publishers; 2004. pp. 147-154.

32 The North Italian Endoscopic Club for the Study and Treatment of Esophageal Varices. Prediction of the first variceal hemorrhage in patients with cirrhosis of the liver and esophageal varices. A prospective multicenter study. N Engl J Med 1988; 319:983-989.

33 Nevens F, Bustami R, Scheys I, Lesaffre E, Fevery J. Variceal pressure is a factor predicting the risk of a first variceal bleeding: a prospective cohort study in cirrhotic patients. Hepatology 1998; 27:15-19.

34 Lebrec D, De Fleury P, Rueff B, Nahum H, Benhamou JP. Portal hypertension, size of esophageal varices, and risk of gastrointestinal bleeding in alcoholic cirrhosis. Gastroenterology 1980; 79:1139-1144.

35 Gluud C, Henriksen JH, Nielsen G. Prognostic indicators in alcoholic cirrhotic men. Hepatology 1988; 8:222-227.

36 Vorobioff J, Groszmann RJ, Picabea E, Gamen M, Villavicencio R, Bordato J, et al. Prognostic value of hepatic venous pressure gradient measurements in alcoholic cirrhosis: a 10-year prospective study. Gastroenterology 1996; 111:701-709.

37 Pascal JP, Cales P. Propranolol in the prevention of first upper gastrointestinal tract hemorrhage in patients with cirrhosis of the liver and esophageal varices. N Eng J Med 1987; 317:856-861.

38 Villanueva C, López-Balaguer JM, Aracil C, Kolle L, González B, Miñana J, et al. Maintenance of hemodynamic response to treatment for portal hypertension and influence on complications of cirrhosis. $J$ Hepatol 2004; 40:757-765.

39 Zipprich A, Winkler M, Seufferlein T, Dollinger MM. Comparison of balloon vs. straight catheter for the measurement of portal hypertension. Aliment Pharmacol Ther 2010; 32:1351-1356.

40 Maleux G, Willems E, Fieuws S, Heye S, Vaninbroukx J, Laleman W, et al. Prospective study comparing different indirect methods to measure portal pressure. J Vasc Interv Radiol 2011; 22:1553-1558.

41 Villanueva C, Albillos A, Genescà J, Abraldes JG, Calleja JL, Aracil C, et al. Development of hyperdynamic circulation and respons to B-blockers in compensated cirrhosis with portal hypertension. Hepatology 2016; 63:197.

42 Aquilar-Olivos N, Motola-Kuba M, Candia R, Arrese M, MéndezSánchez N, Uribe M, Chávez-Tapia NC. Hemodynamic effect of carvedilol vs. propranolol in cirrhotic patients: systematic review and metaanalysis. Ann Hepatol 2014; 13:420-428.

43 Reiberger T, Ulbrich G, Ferlitsch A, Payer BA, Schwabl P, Pinter M, et al. Vienna Hepatic Hemodynamic Lab. Carvedilol for primary prophylaxis of variceal bleeding in cirrhotic patients with haemodynamic non-response to propranolol. Gut 2013; 62:1634-1641.

44 Garcia-Pagan JC, Feu F, Bosch J, Rodés J. Propranolol compared with propranolol plus isosorbide-5-mononitrate for portal hypertension in cirrhosis. A randomized controlled study. Ann Intern Med 1991; 114:869-873.

45 Pietrosi G, D'Amico G, Pasta L, Patti R, Vizzini G, Traina M. Isosorbide mononitrate (IMN) with nadolol compared to nadolol alone for prevention of first bleeding in cirrhosis. A double blind placebo-controlled randomized trial. J Hepatol 1999; 30 (Suppl 1):66

46 Garcia-Pagan JC, Morillas R, Banares R, Albillos A, Villanueva C, Vila C, et al. Spanish Variceal Bleeding Study Group. Propranolol plus placebo versus propranolol plus isosorbide-5-mononitrate in the prevention of a first variceal bleed: a double-blind RCT. Hepatology 2003; 37: 1260-1266. 